\title{
Naming versus matching in color constancy
}

\author{
JIMMY M. TROOST and CHARLES M. M. DE WEERT \\ Nijmegen Institute for Cognition Research and Information Technology, University of Nijmegen \\ Nijmegen, The Netherlands
}

\begin{abstract}
In this paper, a replication of the color-constancy study of Arend and Reeves (1986) is reported, and an alternative method is presented that can be used for the study of higher order aspects of color constancy, such as memory, familiarity, and perceptual organization. Besides a simultaneous presentation of standard and test illuminants, we also carried out an experiment in which the illuminants were presented successively. The results were similar to Arend and Reeves's; however, in the object-matching condition of the successive experiment, we found an overestimation, instead of an underestimation, of the illuminant component. Because the results of matching experiments are difficult to interpret, mainly due to their sensitivity to instruction effects, we introduced another type of color-constancy task. In this task, subjects simply named the color of a simulated patch. It was found that, by applying such a task, a reliable measure of the degree of identification of object color can be obtained.
\end{abstract}

Color constancy is the phenomenon that the color appearance of objects is invariant, notwithstanding variations in illumination. In real-life situations, variations in illumination occur very often. For example, the spectral composition of daylight changes with the weather, the season, and the time of the day. The artificial lights in our environment have many different spectral characteristics as well. In all these cases, the visual system tends to perceive objects with constant colors.

Color constancy poses a problem, because the object component and the illuminant component of the light that reaches the eye are not separately available to the observer. In recent attempts to model color constancy (e.g., Grossberg \& Todorović, 1988; Land, 1977, 1986; Werner $\&$ Walraven, 1982), the crucial question has often been, How does the visual system succeed in taking into account the variations in illumination? Because color constancy in these models is explained by an automatic elimination of the illuminant component by adaptation and lateral inhibition, these models represent sensory explanations. Color-constancy models in artificial vision that try to recover reflectance, and illuminant spectra (e.g., Dannemiller, 1989) also rely on automatic elimination of the illumination component (for a discussion of the relevance of these models to human perception, see Jameson \& Hurvich, 1989, and Troost \& de Weert, 1991). Traditionally, there has been a controversy between sensory (Hering, 1874/1964; von Kries, 1905) and cognitive explanations (Helmholtz, 1867/1962; Katz, 1911/1935; Koffka, 1935) for color constancy (for a review, see Beck, 1972).

The authors wish to thank Adam Reeves and three anonymous reviewers, as well as Jaap Bastiaansen, Noud van Kruysbergen, and Frans Verstraten for thoughtful discussion and constructive comments on earlier versions of this paper. Correspondence should be addressed to Jimmy M. Troost, NICI, University of Nijmegen, P.O. Box 9104 , 6500 HE Nijmegen, The Netherlands (e-mail:troost@psych.kun.nl).
Sensory explanations of color constancy are mechanistic; informational cues to the illuminant are not taken into account. It is implicitly assumed that the visual system does not even notice differences in illumination. On the other hand, cognitive explanations emphasize that both illuminant and object components need to be separately available somewhere in the visual system. In fact, the ability of subjects to make accurate estimations of illuminants (Beck, 1959, 1961) or the perception of illumination as separate from the object due to the figural organization of a scene (Gilchrist, 1980; Gilchrist, Delman, \& Jacobsen, 1983) may be considered as support for a more cognitive point of view.

For the sake of clarity, by cognition, we refer to structural properties of the visual system that reveal the abstract perceptual organization of a scene, such as implemented decision rules in pattern recognition (e.g., Leeuwenberg, 1982). We certainly do not mean conscious reasoning or willingness to see.

In this paper, it will be shown that neither purely sensory nor purely cognitive explanations provide satisfying descriptions of the phenomenon of color constancy (see Part 1). Ideas from the research area of categorical perception will be used to formulate an alternative type of measurement of color constancy that incorporates both sensory and cognitive elements (see Part 2).

\section{PART 1: SENSORY VERSUS COGNITIVE COLOR CONSTANCY}

It is possible to create a sensory and a cognitive colorconstancy task with one and the same experimental setting by using different instructions. Contrary to sensory theories, cognitive theories emphasize the importance of separability - that is, separate perceptions of illuminant and object. In short, our theory is that if subjects are presented with an asymmetric matching task using a stimu- 
lus configuration that does not meet this separability by its figural organization, they will base their color matches solely on sensory information. In a cognitive task, subjects can be given additional information about the construction of the stimulus configuration that enables an interpretation in terms of illuminants and real objects. In this case, matches will be affected by both sensory information and information about the separation of illuminant and object that is explicitly referred to.

Arend and Reeves (1986), using the procedure in which the illumination conditions were spatially separated, found an instruction effect. In this paper, two replications of Arend and Reeves's instruction-effect study will be reported. The first one, being a simultaneous asymmetric matching task in which subjects made adjustments of the achromatic locus, was an actual replication. The second one was a variant in the temporal domain-that is, a successive asymmetric matching experiment. The rationale behind the successive version of the experiment was (1) that it makes more sense to use situations in which one and the same object, or set of objects, are at one place at a time, and (2) that the light illuminating an object generally varies in time.

To test the effect of indirect cues to the illumination on color-constancy matches, the subjects were given an instruction that forced them to extract indirect cues to the illuminant from the stimulus configuration. This condition will be called the object-matching condition. Because the illuminant itself was not visible, these cues are referred to as indirect ones. The illuminant chromaticity was represented by the average color in the stimulus configurations. In the exact-matching condition, no reference to illuminants was made in the instructions.

To summarize, in the exact-matching condition, colorconstancy matches result from sensory processes only, since no additional information is given. In the objectappearance condition, reference is made to illuminants and objects, so the matches are also supposed to be influenced by this separability.

\section{Experiment 1A Simultaneous Matching}

\section{Method}

Subjects. Fourteen undergraduate students in psychology, with normal or corrected-to-normal vision and no color deficiencies, participated as subjects in Experiment $1 \mathrm{~A}$, for which they received course credits. They were naive as to the purpose of the experiment.

Equipment. Stimuli were presented on a high-resolution (1,280X 1,024 pixels) CRT monitor with RGB input connected to an Apollo DN4000 workstation. Chromaticity coordinates of the three phosphors were: $\mathrm{R}, x=0.618, y=0.350 ; \mathrm{G}, x=0.280, y=0.605$; $\mathrm{B}, x=0.152, y=0.063$. It was possible to present 256 colors simultaneously. Each of the colors could have one of $256 \times 256 \times 256$ different RGB combinations.

In the matching experiment, the subjects could control the matching field by means of a mouse, whose position on an $x, y$ table corresponded with a point in the $u, v$-chromaticity diagram. The $u, v$ chromaticity diagram was chosen because it represents perceptual distances more accurately than does the $x, y$-chromaticity diagram (e.g., see Bartleson, 1980). The relation between $x, y$-chromaticity coordinates and $u, v$-chromaticity coordinates is given by

$$
u=4 x /(-2 x+12 y+3), v=6 y /(-2 x+12 y+3) .
$$

The CRT monitor was placed in a darkened room (i.e., no light entered the room from outside and the walls had black wallpaper). The only light came from the CRT monitor. At a viewing distance of $90 \mathrm{~cm}$, the monitor screen subtended a visual angle of $24^{\circ}$ in the horizontal direction and $19^{\circ}$ in the vertical direction.

Stimuli. At a viewing distance of $90 \mathrm{~cm}$, the subjects were presented two configurations of simulated patches against a neutral background. The left configuration was illuminated by the standard illuminant; the right configuration was illuminated by the test illuminant. The middle hexagons in the standard and test configurations served as the target and matching fields, respectively (see Figure 1). The visual angle of one hexagon was $1.4^{\circ}$ and $1.5^{\circ}$ in the horizontal and vertical directions, respectively. Visual angles of the whole configuration of hexagons were $7^{\circ}$ in the horizontal direction and $6.3^{\circ}$ in the vertical direction. The distance in visual angle between the two sets of hexagons was $9^{\circ}$. The colors in both configurations were simulations of the same set of papers under two different illuminants. Three illuminants making six different pairs were considered. Chromaticities of the illuminants in $u, v$ coordinates were: Iluminant $1, u=0.1977, v=0.3123$; Illuminant $2, u=$ $0.2161, v=0.3239$; Illuminant $3, u=0.2354, v=0.3363$. (To get an impression of possible chromaticities of daylight, see Table 1.) The illuminant chromaticity was represented by the average color of the surrounding hexagons. The 18 different surrounding colors were equidistant $(0.035 u, v$ units) to the illuminant chromaticity under the first illuminant. This set of chromaticities was "von Kries transformed" to obtain corresponding chromaticities under the other illuminants (see Appendix). The average color of both the inner ring and the outer ring of hexagons was equal to the illuminant chromaticity. The average luminance of the surrounding colors was $7.5 \mathrm{~cd} / \mathrm{m}^{2}$, and the background luminance was set to $2.5 \mathrm{~cd} / \mathrm{m}^{2}$. Matches had to be made of the left target color at $10.0 \mathrm{~cd} / \mathrm{m}^{2}$. The chromaticity of the target was equal to the standard illuminant chromaticity.

Procedure. The subjects were made familiar with the response apparatus for 10-15 min. After this period, the subjects were instructed either to reproduce the left middle hexagon as precisely as

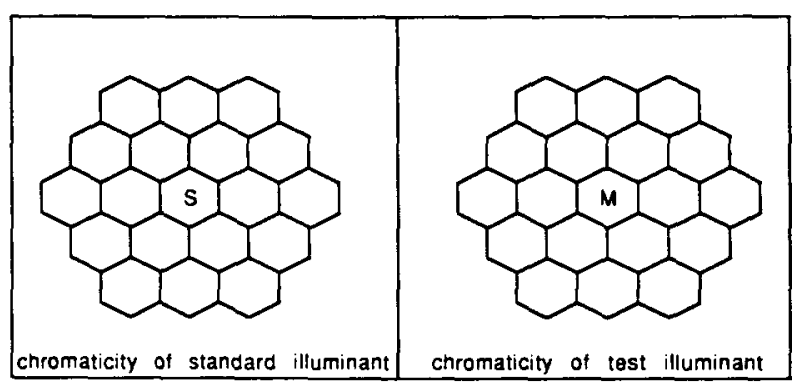

Figure 1. Stimulus used in the simultaneous-matching experiments. The left middle hexagon served as the standard field (S); the right middle hexagon served as the matching field (M). The background chromaticities, as well as the average chromaticities of the surrounding hexagons, were equal to the standard illuminant chromaticity (left) and the test illuminant chromaticity (right). The subject could adjust the chromaticity of the match field on the CRT monitor with the aid of a mouse. 
Table 1

The u,v-Chromaticity Coordinates for Four Phases of Daylight

\begin{tabular}{lcc}
$\begin{array}{c}\text { Corresponding } \\
\text { Color Temperature } \\
\text { (in degrees kelvin) }\end{array}$ & $u$ & $v$ \\
\hline $\mathbf{4 0 0 0}$ (sunlight) & 0.224 & 0.337 \\
$\mathbf{6 5 0 0}$ (overcast sky) & 0.198 & 0.312 \\
10000 (cloudy sky) & 0.186 & 0.295 \\
$\mathbf{2 5 0 0 0}$ (blue sky) & 0.180 & 0.275 \\
\hline
\end{tabular}

Note-The corresponding color temperature equals the temperature of a blackbody radiator having the same chromaticity as daylight.

possible or to interpret the two configurations as real surfaces and to make an object match. In the object-match condition, the subjects received some brief general information about object colors and illuminants to demonstrate that the color of objects is partly determined by the light source. In fact, a light dimmer and some gray patches of paper were used for this purpose. An abbreviated version of the (written) object-appearance instructions is given below.

What you see on the color display is a large sheet of gray paper. The patches in the left configuration are the same as the patches in the right configuration. The differences in color are caused by two different illuminants. Your task is to imagine what the target field will look like if you put it under the test illuminant. Adjust the color of the matching field accordingly.

In the second session, the subjects got the other instruction. In both conditions, the subjects were instructed to switch their gazes between the standard and the test configuration regularly for $1-2 \mathrm{sec}$. Each of the six illuminant pairs was presented 10 times, so $6 \times 10$ responses per subject were registered. If the subject was satisfied with an adjustment, he or she pushed a button and the response was registered; the next trial was given after $2,500 \mathrm{msec}$. The order of the pairs of illuminants was random. In each trial, the starting chromaticity of the match field was randomly selected from the chromaticity diagram. Each session lasted about $1 \mathbf{h}$.

\section{Experiment 1B Successive Matching}

\section{Method}

Subjects. Eight undergraduate students in psychology, with normal or corrected-to-normal vision and no color deficiencies, participated as subjects in Experiment 1B, for which they received course credits. None of these subjects had participated in Experiment $1 \mathrm{~A}$, and all were naive as to the purpose of the experiment.

Equipment. The equipment used in Experiment 1B was the same as that used in Experiment $1 \mathrm{~A}$.

Stimuli. At a viewing distance of $90 \mathrm{~cm}$, the subjects were presented a standard configuration of simulated patches against a neutral background illuminated by the standard illuminant. Subsequently, the test configuration could be retrieved. In fact, this was the same configuration of simulated patches as in the standard, but now under the test illuminant. The colors in both configurations were simulations of the same surfaces under different illuminants. The middle hexagons in the standard and test configurations served as the target and matching fields, respectively (see Figure 2). Visual angles of one hexagon and the whole set of hexagons were the same as in Experiment $1 \mathrm{~A}$.

The chromaticity of the standard illuminant was $u=0.1973, v=$ 0.3139 . Chromaticities of the four test illuminants in $u, v$ coordinates were: (1) $u=0.2350, v=0.3136$; (2) $u=0.1595, v=0.3143$; (3) $u=0.1969, v=0.3499$; (4) $u=0.1972, v=0.2784$. The illuminant chromaticity was represented by the average color of the surrounding hexagons, as was the background color. As in Experiment $1 \mathrm{~A}$, the 18 different surrounding colors were equidistant
(0.035 $u, v$ units) to the illuminant chromaticity and the background luminance; average luminance of the surrounding colors and target luminance were set to $2.5,7.5$, and $10 \mathrm{~cd} / \mathrm{m}^{2}$, respectively. The set of chromaticities under the standard illuminant was "von Kries transformed" to obtain corresponding chromaticities under the other illuminants (see Appendix). The average color of both the inner ring and the outer ring of hexagons was equal to the illuminant chromaticity. The subjects only had to match the color. Five target colors were used. Their chromaticities were: (1) yellow, $u=0.2072, v=$ 0.3526 ; (2) red, $u=0.2372, v=0.3165$; (3) purple, $u=0.2121$, $v=0.2767$; (4) blue, $u=0.1665, v=0.2884$; (5) green, $u=$ $0.1635, v=0.3353$.

Procedure. After a 10- to 15-min period to familiarize themselves with the response apparatus, the subjects read the instructions from the screen. In the exact-matching condition, they had to reproduce the middle hexagon from the standard configuration as precisely as possible in the test configuration.

In the simultaneous experiment, it became clear that the objectmatching condition was much more difficult than the exact-matching condition. Therefore, the object-matching condition was always presented in the second session in this experiment. After some brief general information about objects and illuminants was given to the subjects, the experiment was started. The subjects had to interpret the two configurations as real surfaces. The object-matching instructions were the same as those used in the simultaneous-matching experiment.

In all, $5 \times 4 \times 3=60$ stimuli were given, five target colors, four test illuminants, and three repeated measurements. Each trial started with the standard configuration containing one of the five target colors. The subjects were free to move their gazes over the screen. By pushing a button, the standard configuration disappeared and the background color of the test configuration was shown. After $1,000 \mathrm{msec}$, the hexagons were displayed. In each trial, the starting chromaticity of the match field was randomly selected from the chromaticity diagram. By pushing the same button again, the subjects could switch between the two configurations; they were free to switch between the two configurations as much as they found necessary. Contrary to Experiment $1 \mathrm{~A}$, no instructions were given about the duration of viewing one of the two configurations. After each switch between the standard and test configurations, the positions of the surrounding hexagons were changed randomly. This prevented the subjects from picking out a field with a color that resembled the target color in the standard configuration and then matching the color at the corresponding position exactly in the test configuration. If the subject had finished the adjustment, he or she

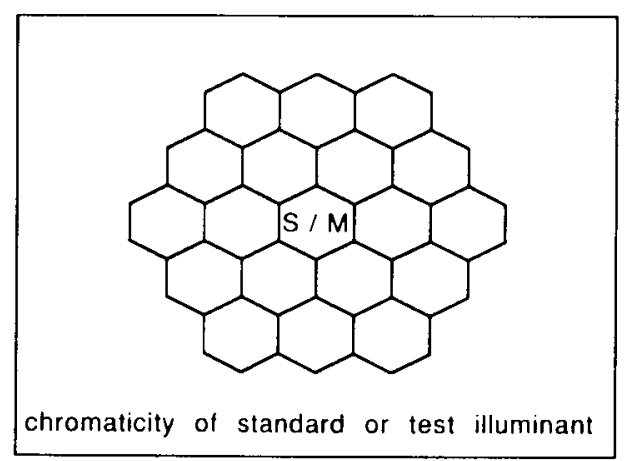

Figure 2. Stimulus used in the successive-matching experiments. The middle hexagon served as either the standard (S) or the matching (M) field. The background chromaticity, as well as the average chromaticity of the surrounding bexigons, was equal to the standard illuminant chromaticity or the test illuminant chromaticity. The subject could adjust the chromaticity of the match field or the CRT monitor with the aid of a mouse. 
pushed another button and the response was registered; the next trial was given after $2,500 \mathrm{msec}$. Each session lasted about $1 \mathrm{~h}$.

\section{Results}

First, an analysis of variance, using a within-subjects design, was performed on the distances between matched colors and target colors for the $u$ - and $v$-chromaticity coordinates separately. A robust instruction effect was found in both the simultaneous and the successive experiments for both dimensions [simultaneous experiment, $F(1,10)=$ $17.32, p<.01, r^{2}=.633$ on the $u$ dimension, and $F(1,10)=7.72, p<.02, r^{2}=.435$ on the $v$ dimension; successive experiment, $F(1,14)=78.49, p<.001, r^{2}=$ .849 on the $u$ dimension, and $F(1,14)=103.20, p<.001$, $r^{2}=.881$ on the $v$ dimension].

Because an analysis of variance is not informative about the degree of color constancy, we defined a twodimensional variant of the Brunswik ratio (Brunswik, 1928). The Brunswik ratio has often been used in brightness constancy and provides a measure of the degree of constancy. In the one-dimensional case, it is defined as

$$
\text { br }=(\mathrm{O}-\mathrm{S}) /(\mathrm{E}-\mathrm{S}) \text {, }
$$

where $O$ is the observed match, $S$ is the standard, and $E$ is the expected match. A ratio of 0 represents a complete lack of constancy; a ratio of 1 represents perfect constancy (prf in subscripts). Because color matching occurs in two dimensions ( $u$ - and $v$-chromaticity coordinates), we defined a two-dimensional Brunswik ratio as follows:

$$
\begin{aligned}
& d_{\mathrm{obs}}=\sqrt{\left(u_{\mathrm{obs}}-u_{\mathrm{std}}\right)^{2}+\left(v_{\mathrm{obs}}-v_{\mathrm{std}}\right)^{2}}, \\
& d_{\mathrm{prf}}=\sqrt{\left(u_{\mathrm{prf}}-u_{\mathrm{std}}\right)^{2}+\left(v_{\mathrm{prf}}-v_{\mathrm{std}}\right)^{2}}, \\
& \mathrm{br}=d_{\mathrm{obs}} / d_{\mathrm{prf}},
\end{aligned}
$$

where $d_{\mathrm{obs}}$ and $d_{\mathrm{prf}}$ are the euclidean distances between the location of the standard $\left(u_{\mathrm{std}}, v_{\mathrm{std}}\right)$ and the observed match $\left(u_{\mathrm{obs}}, v_{\mathrm{obs}}\right)$, and between the standard and the perfect color-constancy match $\left(u_{\mathrm{prf}}, v_{\mathrm{prf}}\right)$, respectively. The Brunswik ratio is defined as the ratio of these distances. This measure is similar to the constancy index that was

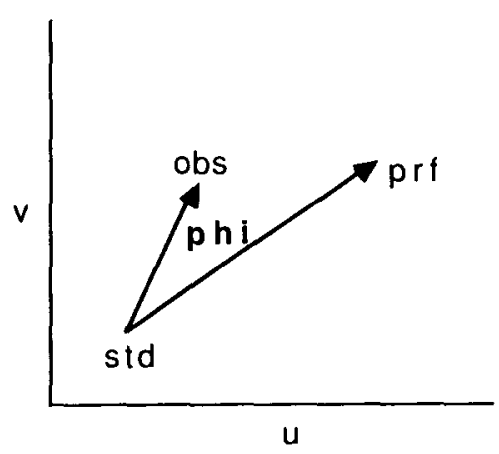

Figure 3. Graphical presentation of the parameter phi. Phi is defined as the angle between the line connecting the standard chromaticity (std) and the observed chromaticity (obs), and the line connecting the standard chromaticity and the perfect color-constancy match (prf). The angular deviation phi is defined in $u, v$ space.
Table 2

\begin{tabular}{|c|c|c|c|c|}
\hline \multirow[b]{2}{*}{ Iluminant } & \multicolumn{2}{|c|}{ br } & \multicolumn{2}{|c|}{ phi } \\
\hline & $M$ & $S D$ & $M$ & $S D$ \\
\hline \multicolumn{5}{|c|}{ Exact-Matching Condition } \\
\hline 12 & 0.53 & 0.07 & -1 & 10 \\
\hline 13 & 0.41 & 0.09 & 3 & 19 \\
\hline 21 & 0.46 & 0.09 & 1 & 21 \\
\hline 23 & 0.47 & 0.23 & -3 & 28 \\
\hline 31 & 0.42 & 0.10 & 2 & 19 \\
\hline 32 & 0.48 & 0.11 & 4 & 10 \\
\hline \multicolumn{5}{|c|}{ Object-Matching Condition } \\
\hline 12 & 0.87 & 0.16 & 4 & 15 \\
\hline 13 & 0.79 & 0.18 & 4 & 13 \\
\hline 21 & 0.79 & 0.21 & 1 & 19 \\
\hline 23 & 0.90 & 0.17 & 7 & 16 \\
\hline 31 & 0.77 & 0.20 & 1 & 17 \\
\hline 32 & 0.81 & 0.25 & -2 & 14 \\
\hline
\end{tabular}

Means and Standard Deviations of br and phi (in Degrees) Values per Condition and per Illuminant Pair of the Simultaneous-Matching Experiment

Note-Standard deviations were calculated over subjects.

Table 3

\begin{tabular}{|c|c|c|c|c|}
\hline \multirow[b]{2}{*}{ Iluminant } & \multicolumn{2}{|c|}{ br } & \multicolumn{2}{|c|}{ phi } \\
\hline & $M$ & $S D$ & $M$ & $S D$ \\
\hline \multicolumn{5}{|c|}{ Exact-Matching Condition } \\
\hline $\mathbf{R}$ & 0.38 & 0.14 & 3 & 28 \\
\hline B & 0.39 & 0.14 & -1 & 19 \\
\hline $\mathrm{G}$ & 0.41 & 0.37 & 7 & 25 \\
\hline $\mathbf{Y}$ & 0.49 & 0.15 & 8 & 13 \\
\hline \multicolumn{5}{|c|}{ Object-Matching Condition } \\
\hline $\mathbf{R}$ & 1.51 & 0.52 & 4 & 16 \\
\hline B & 1.35 & 0.61 & 6 & 23 \\
\hline G & 1.29 & 0.41 & -13 & 38 \\
\hline $\bar{Y}$ & 1.35 & 0.53 & -7 & 21 \\
\hline
\end{tabular}

Means and Standard Deviations of br and phi (in Degrees) Values per Condition and per Test Iluminant of the Successive-Matching Experiment

Note-Standard deviations were calculated over subjects.

recently used by Arend, Reeves, Schirillo, and Goldstein (1991), the only difference being that we took the distances from the standard, whereas they took distances from the perfect color-constancy match. Unfortunately, neither our Brunswik ratio nor Arend et al.'s constancy index gives complete information about the degree of color constancy. In the two-dimensional color-matching case, an angular deviation must also be defined:

$$
\begin{aligned}
& u_{1}=u_{\mathrm{obs}}-u_{\mathrm{std}}, \\
& v_{1}=v_{\mathrm{obs}}-v_{\mathrm{std}}, \\
& u_{2}=u_{\mathrm{prf}}-u_{\mathrm{std}}, \\
& v_{2}=v_{\mathrm{prf}}-v_{\mathrm{std}}, \\
& \mathrm{phi}=\operatorname{sign}\left(u_{1} v_{2}-u_{2} v_{1}\right) \arccos \left(u_{1} u_{2}+v_{1} v_{2}\right) /\left(d_{\mathrm{obs}} d_{\mathrm{prf}}\right) .
\end{aligned}
$$

See Figure 3 for the graphical interpretation of the angular deviation phi.

In Tables 2 and 3, the br and phi are listed for the exactand object-matching conditions for Experiments $1 \mathrm{~A}$ and 


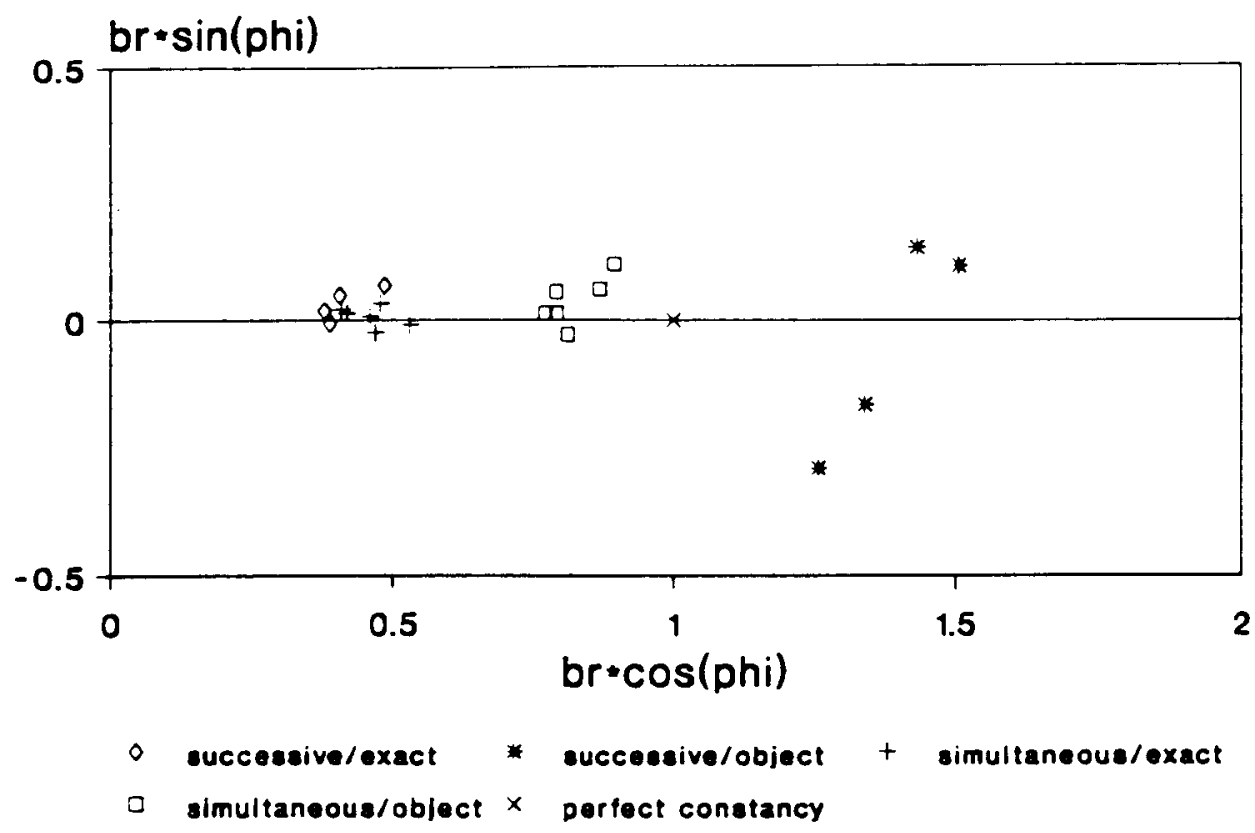

Figure 4. Plot of the data of the simultaneous- and successive-matching experiments in polar coordinates. Each symbol represents one illuminant level (note that there were six in the simultaneous experiment and four in the successive experiment). The length of the line drawn from the origin to a symbol is equal to the Brunswik ratio, br, in the corresponding condition, whereas the angle of this vector with the abscissa is equal to the angular deviation, phi (see also Tables 2 and 3).

1B. For the exact-matching condition, the overall br and phi were found to be 0.46 and $0.9^{\circ}$, respectively, in the simultaneous experiment, and 0.41 and $4.4^{\circ}$, respectively, in the successive experiment; in the object-matching condition, experiment br was 0.82 and phi was $2.4^{\circ}$ in the simultaneous experiment, and br was 1.51 and phi was $-4.7^{\circ}$ in the successive experiment. Perfect color constancy is achieved if $b r=1$ and $p h i=0$. So, if phi does not differ much from 0 , as was the case in all experimental conditions, a br smaller than 1 indicates underconstancy, whereas a br larger than 1 indicates overconstancy. In Figure 4 , the results of each experimental condition are displayed in polar coordinates.

\section{Discussion}

All subjects saw color differences when comparing the standard with the test, and some of them reported they had difficulties with it. If color constancy was perfectly achieved by sensory processes only, color differences between standard and test configurations should not be perceived in either the exact-matching or the objectappearance condition. Similar to Arend and Reeves's (1986) results, the exact-matching condition revealed a relatively low degree of color constancy in both the simultaneous and the successive experiments (simultaneous, br $=0.46$, phi $=0.9^{\circ}$; successive, br $=0.41$, phi $=$ $4.4^{\circ}$ ). These findings may be considered as evidence for the view that color constancy is only partly determined by chromatic adaptation and lateral inhibition. Since no refer- ence was made to the separation of illuminant and object components in the exact-matching condition, the degree of color constancy found in this condition is an estimation of the performance of sensory processes. However, because the subjects freely switched between the standard and test configurations (although they were instructed to switch their gazes every 1-2 sec in the simultaneousmatching experiment), it is possible that the contribution of slow adaptation processes is underestimated. However, tasks such as these do not seem to activate all processes involved in the achievement of color constancy, they only incorporate chromatic induction.

The results of the object-appearance condition must be judged with care. Instead of matching what one sees (exact matching), the subjects had to match what they should see. Therefore, these results do not point to a difference in sensation but rather to the ability of subjects to separate illuminant and object components and adjust their matches in accordance with their estimations. We think that since, in the object-matching condition, subjects strongly rely on indirect knowledge about objects and illuminants that have to be made explicit, the uncertainty of the matches is higher than in the exact-matching condition. Thus, in our view, the differences in br between the object- and exact-matching conditions are caused by different judgments rather than different sensations. If this is the case, it is simply not allowed to relate these results to color constancy as a visual phenomenon only. One important remark must be made, however. The results of both the simultaneous- and the successive-object conditions suggest 
that knowledge about illuminant and objects can strongly influence color judgments; it does not imply that there are differences in sensory data between the two conditions.

A large overcompensation of the illuminant was found in the object-instruction condition of the successive experiment $\left(\mathrm{br}=1.51\right.$, phi $\left.=-4.7^{\circ}\right)$. However, the shifts of the yellow and green colors under the yellow test illuminant were difficult to accomplish because the resulting colors were very close to the borders of the color range of the monitor. Therefore, these data may be biased (if these colors are excluded, then average $\mathrm{br}=1.38$ and average phi $=-2.0^{\circ}$ ). So far, we have not been able to formulate a satisfactory explanation for the fact that, in the object-instruction condition of the successive experiment, we found an overcompensation, whereas in the corresponding condition of the simultaneous experiment we found an undercompensation. This undercompensation, however, is consistent with Arend and Reeves's (1986) findings. One can speculate about the difference in procedure of presenting the standard and test illuminant (successive vs. simultaneous). In the successive task, subjects do not have a direct comparison of the illuminant, so any color matches are based on representations of the color they saw under the standard illuminant and the illuminant component itself. Color representations, or memory colors, are known to shift in time to prototypical colors that appear more saturated (e.g., Pijnenborgh, 1987). As a result of this shift, the illuminant component may be overestimated in the successive experiment.

To summarize, neither of the two conditions provides convincing color-constancy data. Contrary to the objectappearance condition, the exact-matching condition has the advantage of more closely registering the product of visual sensation. Unfortunately, it is confined to the sensory part of color constancy only. The advantage of the object-appearance condition is that sensory information and the separation of illuminant and object components are integrated in the subjects' responses. If only the results are considered, the simultaneous-object-appearance condition, as opposed to the successive version, gives quite convincing evidence for color constancy. However, in the ideal case, one would like to have a color-constancy task that registers the product of visual perception in which the separation of illuminant and object components is met more naturally than is the case in matching tasks. For this purpose, we tried an alternative color-constancy task based on color naming.

\section{PART 2: CATEGORICAL COLOR CONSTANCY}

The rationale for this proposal stems mainly from the next consideration. Usually, color constancy is described in a way that points to the ability of the visual system to eliminate the illuminant component from the light reaching the eye. It is a logical consequence that if color constancy is described as the result of a compensatory pro- cess, methods are used that can give detailed information about the success of such a process. Until now, the matching method has been used most often because very accurate results about the degree of compensation can be obtained (as expressed, for example, by the Brunswik ratio that was described in Part 1). In our view, the relatively low degree of color constancy reported in this paper (exact-matching conditions) and elsewhere (e.g., Arend \& Reeves, 1986; Valberg \& Lange-Malecki, 1990) implies the absence of appropriate compensatory mechanisms. However, unlike Valberg and Lange-Malecki (1990), we do not wish to conclude that color constancy is a nonexistent phenomenon.

If color constancy would be described from a more natural perspective, it can be argued that the purpose of color constancy is to prevent erroneous object-color perceptions due to variations in illumination. Color constancy would exist as long as an object is not assigned a different color under different illuminant conditions. Such a description does not deny compensatory mechanisms, such as chromatic adaptation and lateral inhibition, to be operative, but it poses less extreme demands on the performance of these processes. Or, as Jameson and Hurvich $(1989$, p. 7) argue, color shifts resulting from different illuminations are not so great to prevent object identification by color, because these shifts remain within colorcategory boundaries, which would not be the case without compensatory mechanisms such as chromatic adaptation and lateral inhibition. Recall that according to the common discounting-the-illuminant definition of color constancy, these mechanisms are implicitly supposed to perfectly eliminate the illuminant component from the light reaching the eye. From both the exact-matching data presented in the previous part and Arend and Reeves's (1986) data, it appears that these mechanisms can only account for about $40 \%$ of the color shift necessary to reach a perfect color-constancy match. However, although these results seem to indicate that many errors are made in object-color perception, this is certainly not the impression one gets under ordinary viewing conditions: every object retains its color despite changes in illumination. If Jameson and Hurvich's suggestion about color-category boundaries is taken into account, the discrepancy between daily experience, on the one hand, and the relatively poor performance of chromatic adaptation and lateral inhibition processes, on the other hand, may very well be due to the categorical character of object-color perception. Although the visual system is very sensitive to color differences and can discriminate a very large number of colors, colors are perceptually grouped into discrete categories (e.g., Bornstein, 1987; Boynton, Fargo, Olson, \& Smallman, 1989; Uchikawa, Uchikawa, \& Boynton, 1989). Consequently, color constancy should be studied in an identification task rather than in a matching task, which is more appropriate for discrimination phenomena. Harnad (1987) refers to identification tasks as tasks in which labels have to be assigned to stimuli. So, applying 
the terminology of Harnad to the aforementioned description, color constancy should be studied as an identification phenomenon.

In Experiment 2, the subjects had to name the color of simulated object patches against a simulated neutral background under different illuminations. In this task, the success of identification - that is, assigning the same color name to the same patch under different illuminants-provides a measure of color constancy.

\section{Experiment 2: Color Naming}

\section{Method}

Subjects. Thirty undergraduate students in psychology, with normal or corrected-to-normal vision and no color deficiencies, participated as subjects in Experiment 2, for which they received course credits. They were naive as to the purpose of the experiment.

Equipment. The equipment used in Experiment 2 was the same as that used in Experiment 1A, except that now the mouse was used to position a cursor on the CRT monitor.

Stimuli. At a viewing distance of $90 \mathrm{~cm}$, the subjects were shown a colored circle, subtending a visual angle of $1.6^{\circ}$. The basic color set contained 24 hue $\times 3$ saturation $\times 2$ luminance levels $=144$ chromaticities of simulated patches, enabling us to investigate a wide range of colors. This set was chosen to represent a sufficiently large range of colors under the white illuminant (that approximated CIE illuminant D65). Next, the chromaticities of each of the 144 simulated patches was calculated according to the color shift due to the red, green, yellow, and blue illuminants (see Figure 5). Thus, each of the 144 simulated patches could have five different chromaticities, depending on the illuminant.

The chromaticities of the illuminants were: white, $u=0.1973$, $v=0.3138$; red, $u=0.2273, v=0.3138$; green, $u=0.1673$, $v=0.3138$; yellow, $u=0.1973, v=0.3438$; blue, $u=0.1973$, $v=0.2828$. The chromaticity of the background corresponded to

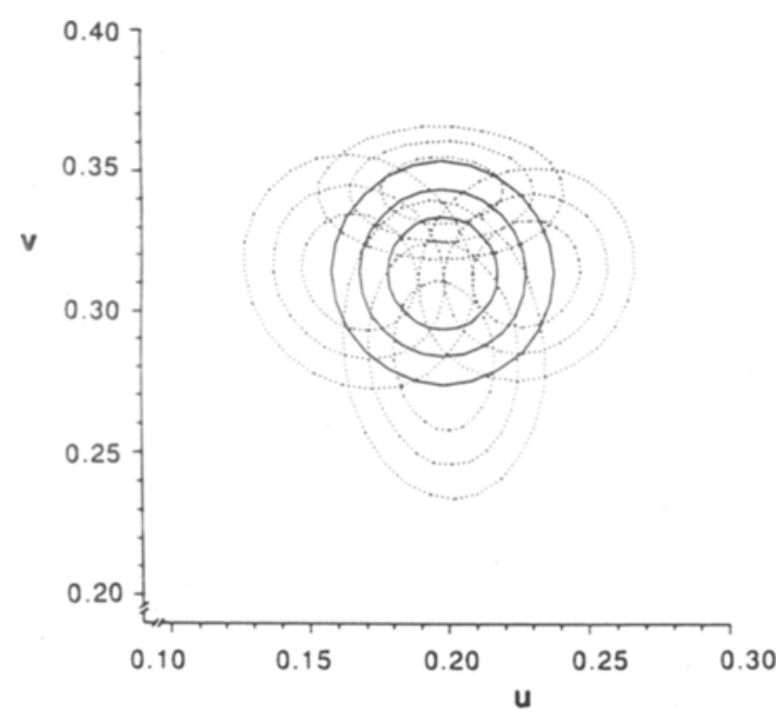

Figure 5. Stimulus set used in the color-naming experiment. On the solid lines, the chromaticities under the white illuminant are found. Fach of the four other sets of three concentric ellipsoids (dashed lines) represent the colors under the test illuminants: (starting at the top and moving clockwise) yellow, red, blue, and green. The chromaticities of the simulated illuminants are located in the center of each set of ellipsoids. the chromaticity of the illuminants. The luminance of the background was $5 \mathrm{~cd} / \mathrm{m}^{2}$. The set of chromaticities under the standard illuminant was "von Kries transformed" to obtain corresponding chromaticities under the other illuminants (see Appendix).

In the low- and high-luminance conditions, color stimuli were displayed at 2.5 and $10 \mathrm{~cd} / \mathrm{m}^{2}$, respectively. The three saturation conditions differed in the distance of the colors to the achromatic locus of the white illuminant $(0.02,0.03$, or $0.04 u, v$ units $)$.

Procedure. During the entire session, a response box containing 12 different monolexemic color names was visible. The color of the lines of the response box and the characters was black. The subjects were instructed to carefully inspect the available response categories. The color names were in Dutch, so we give the English translations: red, green, yellow, blue, orange, pink, brown, white, gray, lilac, and two words for purple (paars and purper), which cannot be appropriately translated. The stimulus color was displayed for $750 \mathrm{msec}$. Next, the subjects had to place the cursor on 1 of the 12 color names and push a mouse button. The next trial followed after $500 \mathrm{msec}$

Nothing was told about illuminants or objects. The subjects were simply instructed to choose the most appropriate color name. They were told not to wonder too long about their choices. Accuracy and speed were stressed, although it was mentioned that reaction times were not registered. No information about the response categories was given by the experimenter. The subjects were instructed to name the stimulus colors using their own naming strategy.

After a few minutes of practice, the experiment started. In all, $5 \times 144$ stimuli, divided over five blocks separated by a short pause, were presented to each of the 30 subjects. First, the color names under the white illuminant were obtained. Next, each of the four other illuminants was considered.

\section{Results}

For each subject, the color name given to each simulated patch under a standard illuminant served as the standard color name. The standard illuminant was one of the five illuminants used in Experiment 2. Next, the standard color name was compared with each color name given to the corresponding simulated patch under one of the four other illuminants. The number of violations or deviations from the standard color name was counted. Thus, per simulated patch, the number of violations could range from zero to four, indicating perfect to imperfect identification over illuminants, respectively. For each subject, $5 \times 144$ scores were obtained, 144 per standard illuminant. The categories for purple ("paars" and "purper") and lilac were pooled in this analysis, because a first inspection of the data revealed that they overlapped each other and were used interchangeably between and within subjects. The pooling had a marginal effect on the data.

Because our primary interest was to discover a general trend regardless of illuminant, hue, or saturation and luminance levels, all data were pooled. In Figure 6 (white bars), the proportional frequencies of the number of violations are displayed. As can be seen from this figure, the distribution of the number of violations is toward perfect identification (no violations occurred in $39.9 \%$ of all observations; $38.3 \%$ if the purple categories are not pooled). This general trend in the distribution of proportional frequencies was found in all conditions. Therefore, color naming under different illuminations is an appropriate identification task to investigate color constancy. 


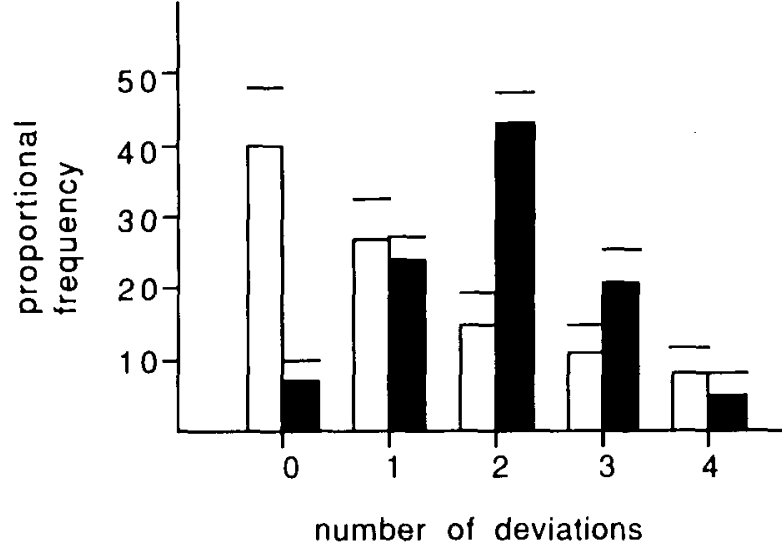

Figure 6. Proportional frequencies of the number of deviations that occurred in the color-constancy condition (white bars) and the control condition (shaded bars) of the color-naming experiment. Color names given under the standard illuminant were used as targets. So, under the four test illuminants, the total number of deviations per simulated patch could vary between zero (perfect identification) and four (no identification). Lines above bars indicate standard deviations.

Presumably, the size of the basic set of color names presented to the subjects affects the number of violations that are made. If subjects can choose only from four color names (e.g., red, green, yellow, and blue), it is obvious that there generally will ive more responses of the same name for a patch under different illuminations. Alternatively, increasing the set size may lead to a decrease of the degree of identification. Therefore, it is important to select the set of color names carefully. In this study, we used a slightly modified version of the color set that was empirically obtained by Uchikawa et al. (1989), who allowed their subjects to give any monolexemic color name that came to mind in response to colored patches. For the moment, we can only point to the set-size problem. Other experiments are in preparation to reveal in more detail the relations between set size and degree of identification.

It might be argued that the relatively high degree of identification is due to the fact that the area of chromaticities assigned to one particular color name (e.g., red) may include many of the corresponding transformed chromaticities under other illuminants. So, if the transformed chromaticities of the patch called red fall in the area of all chromaticities called red, the response is not affected by the simulated illuminant. The high degree of identification would then be a result of an artifact rather than a result of the separation of illuminant information present in the background. We anticipated this bias and included a control condition in the experiment. To estimate this bias, we also presented each of the $5 \times 144$ test chromaticities from Figure 5 against a background having the chromaticity of the white illuminant, rather than the corresponding illuminant chromaticity (located in the center of each of the five sets of three concentric ellipses in Figure 5). Thus, illuminant information was kept constant for all five sets of test chromaticities. Next, we obtained a second set of violations scores by applying the same procedure as described above to the control data. The results are also displayed in Figure 6 (shaded bars). The shape formed by the white bars differs substantially from the shape formed by the shaded bars. For the constantillumination condition, the peak lies at two violations; for the experimental condition, the peak is at zero violations. Therefore, the relatively high degree of identification cannot be ascribed to the fact that the area of chromaticities assigned to one particular color name includes many of the corresponding transformed chromaticities under other illuminants.

To compare the results from the categorization experiment with those of matching experiments, a subsequent analysis was performed on the categorization data. In this analysis, the data were transformed to interval level in order to derive Brunswik ratios (Brunswik, 1928). The procedure to obtain Brunswik ratios from the data naming data will be described below. First, however, we want to emphasize that our only intention is to give an indication of the difference between naming and matching in general. We certainly do not want to demonstrate the superiority of one method over the other.

Because the subjects had to choose from a restricted set of 12 monolexemic color names for each of the 144 simulated patches, it was possible to locate each of these 12 color categories in the $u, v$-chromaticity diagram. All data were pooled over subjects. The locations were calculated by taking the average $u, v$ values of all color stimuli that were called red, green, purple, and so forth. This was done for the standard illuminant and for each of the four test illuminants; thus, five different maps of the location of color names in $u, v$-chromaticity space were obtained. It was then possible to calculate the Brunswik ratio for each color name by following the same procedure as in Part 1. The results are given in Table 4. However, to obtain the Brunswik ratio for each of the 144 simulated patches separately, a second transformation is required.

Table 4

Reconstructed Means and Standard Deviations of br and phi (in Degrees) Values per Monolexemic Color Category Used in the Color-Naming Experiment

\begin{tabular}{lccrrr}
\hline & \multicolumn{2}{c}{ br } & & \multicolumn{2}{c}{ phi } \\
\cline { 2 - 3 } \cline { 5 - 5 } Category & $M$ & $S D$ & & $M$ & $S D$ \\
\hline Blue & 0.75 & 0.09 & & -2 & 6 \\
Brown & 0.55 & 0.14 & & -13 & 14 \\
Gray & 0.33 & 0.14 & 7 & 45 \\
Green & 0.64 & 0.09 & -5 & 8 \\
Lilac & 0.76 & 0.16 & 4 & 14 \\
Orange & 0.71 & 0.15 & -9 & 15 \\
Pink & 0.79 & 0.05 & -1 & 3 \\
Purple* & 0.84 & 0.24 & 0 & 10 \\
Purple $\dagger$ & 0.64 & 0.12 & -4 & 9 \\
Red & 0.88 & 0.11 & -3 & 8 \\
White & 0.36 & 0.13 & -7 & 25 \\
Yellow & 0.60 & 0.09 & -2 & 11 \\
\hline
\end{tabular}

Note-The results were averaged over all illuminant pairs. *The Dutch word paars for purple, which cannot be appropriately translated. †The Dutch word purper for purple, which cannot be appropriately translated. 
Table 5

Reconstructed Means and Standard Deviations of br and phi (in Degrees) Values per Illuminant Pair in the Color-Naming Experiment

\begin{tabular}{cccrrr}
\hline & \multicolumn{2}{c}{ br } & & \multicolumn{2}{c}{ phi } \\
\cline { 2 - 3 } \cline { 5 - 6 } Illuminants & $M$ & $S D$ & & $S$ \\
\hline WR & 1.01 & 0.37 & & -23 & 21 \\
WB & 0.59 & 0.34 & & -34 & 56 \\
WG & 0.68 & 0.28 & & 30 & 42 \\
WY & 1.22 & 0.23 & & 7 & 24 \\
RW & 0.64 & 0.34 & & 51 & 48 \\
RB & 0.46 & 0.27 & & -3 & 55 \\
RG & 0.58 & 0.25 & 14 & 22 \\
RY & 0.90 & 0.28 & & 27 & 21 \\
BW & 1.08 & 0.35 & 12 & 24 \\
BR & 1.00 & 0.32 & & -5 & 12 \\
BG & 0.78 & 0.32 & & 15 & 21 \\
BY & 1.02 & 0.17 & & 5 & 13 \\
GW & 1.09 & 0.47 & & -27 & 28 \\
GR & 0.85 & 0.26 & -17 & 14 \\
GB & 0.71 & 0.35 & -37 & 28 \\
GY & 1.06 & 0.33 & -10 & 19 \\
YW & 0.62 & 0.40 & & -53 & 78 \\
YR & 0.70 & 0.27 & -38 & 32 \\
YB & 0.45 & 0.30 & -27 & 39 \\
YG & 0.47 & 0.26 & 16 & 62 \\
\hline
\end{tabular}

Note-Standard deviations were calculated over the set of 144 simulated patches.

First, for each of the $5 \times 144$ chromaticities, the distribution of color-name responses was determined over subjects. Second, the relative frequencies under one illuminant served as weighting factors that were used to predict a response under another illuminant.
For example, under the red test illuminant, a simulated patch was called yellow by 18 subjects, orange by 6 subjects, and red by 6 subjects. The relative frequencies for yellow, orange, and red are $60 \%, 20 \%$, and $20 \%$, respectively. These numbers were used as weighting factors to predict a response under the green illuminant, for example, as follows. The $u, v$ coordinates were found by calculating the weighted average of the $u, v$ coordinates of the locations of yellow, orange, and red in $u, v$-color space under the green test illuminant. It was then possible to calculate the Brunswik ratio for each of the 144 simulated patches for every illuminant pair used in this experiment.

The $u, v$ coordinates of the standard, predicted, and reconstructed observed colors can be substituted in the formulas for the two-dimensional Brunswik ratio given in Part 1. The average br and phi for each illuminant pair are given in Table 5 and are displayed in Figure 7.

\section{Discussion}

Because only the white illuminant was used as a standard in the matching experiment, only the entries in which the white illuminant is the standard (see Table 5) are valid comparisons. If the white illuminant was used as a standard, the average br was 0.87 and phi was $-5.2^{\circ}$. This is an improvement relative to the results of the exactmatching experiment $\left(\mathrm{br}=0.41\right.$, phi $=4.4^{\circ}$ ), although the variability of both $b r$ and phi is much larger in the naming experiment (see Table 5 and Figure 7).

Actually, a strict comparison between naming and matching is not possible because there are a few differences between the methods-the most important being the fact that a blockwise presentation of illuminant conditions

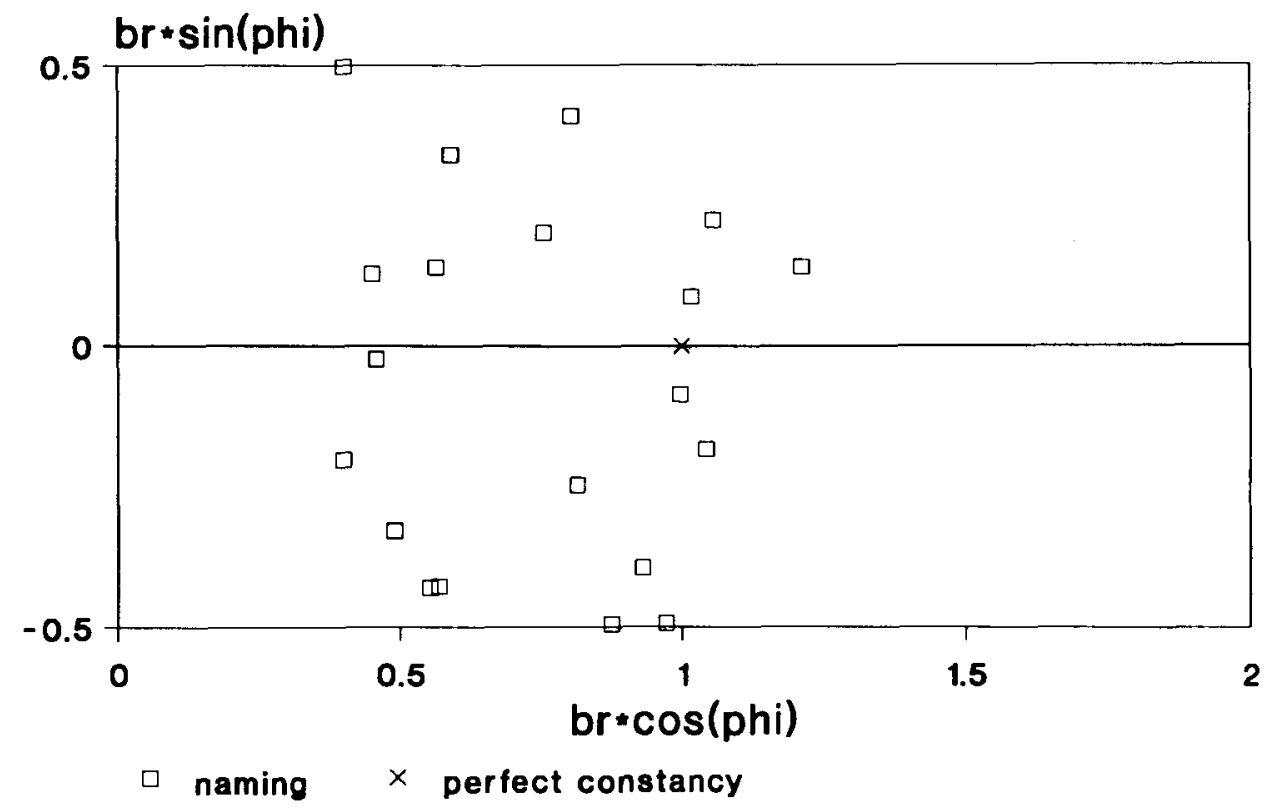

Figure 7. Plot of categorical Brunswik ratios, br, and angular deviations, phi, in polar coordinates. The length of the line drawn from the origin to a symbol is equal to br in the corresponding condition, whereas the angle of this vector with the absecisa is equal to phi. For transformations, see text. Each symbol represents an illuminant pair (see also Table 5). 
was used in the naming experiment, whereas a randomly mixed presentation was used in the matching experiments. This point will be discussed in more detail below. Other differences were: (1) free viewing was possible in both matching experiments, whereas exposures of limited duration $(750 \mathrm{msec})$ were used in the naming experiment; and (2) 18 different colors were presented in the surround in the matching experiments, whereas the only surround color was the one of the background in the naming experiment. Despite these differences, we think it is informative to present the transformation from categorical data to virtual matching data.

An explanation for the difference in average performance can be that the subject's state of adaptation was better controlled in the naming experiment because of the blockwise presentation of the illuminant conditions. Recall that, in the simultaneous-matching experiment, the subjects were instructed to switch their gazes from the one configuration to the other regularly for 1-2 sec. However, such an instruction does not guarantee that the subjects in fact did so. In the successive-matching experiments, no restriction was imposed on the duration of viewing the standard and test configurations. One could argue that, in both matching tasks, the subject's state of adaptation was poorly controlled. In a sense, this is an inevitable artifact of the type of matching experiment in which both standard and test configurations are seen with the same eyes. A switch between standard and test configurations will always change the state of adaptation. Continuously modifying the color of the matching field requires the availability of the target color, whether it be simultaneous or successive, simply because matching takes time, and one wants to avoid distortions that may be addressed to memory. This means that a blockwise presentation of illuminant conditions, as in the naming experiment, is logically impossible. However, there is an alternative way to overcome this dissimilarity problem.

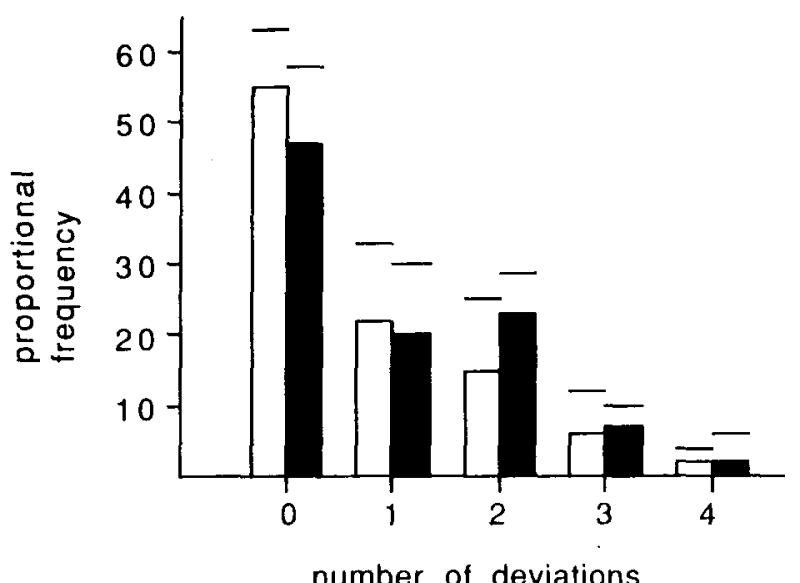

Figure 8. Proportional frequencies of the number of deviations that occurred in the blockwise (white bars) and the randomized illuminant (shaded bars) conditions of the additional color-naming experiment. Lines above bars indicate standard deviations. For explanation, see Figure 7.
Although blockwise matching is impossible, a naming task in which the illuminant conditions are randomized from trial to trial is possible. Now, if one wishes to take an extreme stand and concludes that the difference between naming and exact matching is mainly determined by the different adaptational states, one would predict a considerable change in performance in the randomized condition. But this is not what happens. In Figure 8, the frequency distributions are plotted for a blockwise (white bars) and a randomized (shaded bars) presentation of illuminant conditions in a naming task that is similar to the one described above. Although the difference between the two distributions is in the direction that is expected from an adaptation explanation, it is clear that the difference is not serious.

\section{CONCLUDING REMARKS}

In this paper, an investigation of color-matching and color-naming tasks in the study of color constancy was reported. It was argued that color-matching tasks are not appropriate because they do not allude to the identification aspects of color constancy. Furthermore, it was found that unequivocal responses cannot be obtained using this kind of task because the type of instruction requires a fundamentally different interpretation of the data. As an alternative, a color-naming task was proposed. The number of violations in color naming under different illuminations appeared to be an appropriate measure of color constancy that does not require the separation of illuminants and objects to be explicitly made clear to the subjects.

Finally, we want to give a suggestion for the future study of color constancy. Following Koffka (1935), the separation of illuminant and object components is completely governed by an organizational process that can be thought of as a rule-based decision mechanism that is implemented in the hardware of the visual system. The activity of this mechanism cannot be penetrated by cognitive reasoning, and its outputs are perceptually coercive, although they can be made available to consciousness. In our view, color constancy is not simply the result of an automatic elimination of the illuminant component in the light that reaches the eye, rather it is the product of a mechanism that prevents errors in the identification of objects due to differences in illumination. This new description of color constancy, in which the importance of stable-object perceptions over illuminants is emphasized, requires approaches that model more complex and intelligent behavior than just adaptation and lateral inhibition. Although color categorization is not the solution to color constancy, the method of color naming is an appropriate tool that can be used in the investigation of the rules leading to a separation of illuminant and object components in visual perception.

\section{REFERENCES}

AREND, L., \& ReEves, A. (1986). Simultaneous color constancy. Journal of the Optical Society of America A, 3, 1743-1751.

AREND, L., ReEves, A., SCHIRILlo, J., \& GoldsteIN, R. (1991). Simul- 
taneous color constancy: Papers with diverse Munsell values. Journal of the Optical Society of America A, 8, 661-672.

Bartleson, C. (1980). Colorimetry. In F. Grum \& C. Bartleson (Eds.), Optical radiation measurements (Vol. 2, pp. 33-148). New York: Academic Press.

BECK, J. (1959). Stimulus correlates for the judged illumination of a surface. Journal of Experimental Psychology, 58, 267-274.

BECK, J. (1961). Judgements of surface illumination and lightness. Journal of Experimental Psychology, 61, 368-373.

BECK, J. (1972). Surface color perception. Ithaca, NY: Cornell University Press.

Bornstein, M. (1987). Perceptual categories in vision and audition. In S. Harnad (Ed.), Categorical perception (pp. 287-300). New York: Cambridge University Press.

Boynton, R., Fargo, L., Olson, C., Smallman, H. (1989). Category effects on color memory. Color Research \& Application, 14, 229-234.

BrILL, M., West, G. (1986). Chromatic adaptation and color constancy: A possible dichotomy. Color Research \& Application, 11, 196-204.

BrunswiK, E. (1928). Zur Entwicklung der Albedowahrnehmung. Zeitschriff fur Psychologie, 64, 216-227.

DANNEMILlER, J. (1989). Computational approaches to color constancy: Adaptive and ontogenetic considerations. Psychological Review, 96, 255-266.

GiLchrist, A. L. (1980). When does perceived lightness depend on perceived spatial arrangement? Perception \& Psychophysics, 28 , 527-538.

Gilchrust, A., Delman, S., * Jacobsen, A. (1983). The classification and integration of edges as critical to the perception of reflectance and illumination. Perception \& Psychophysics, 33, 425-436.

Grossberg S., * Todorović, D. (1988). Neural dynamics of 1-D and 2-D brightness perception: A unified model of classical and recent phenomena. Perception \& Psychophysics, 43, 241-277.

HaRnad, S. (Ed.) (1987). Categorical perception. New York: Cambridge University Press.

HeLmHOLTZ, H. vON (1962). Treatise on physiological optics (J. Southall, Ed. and Trans.). New York: Dover. (Original work published 1867)

HerING, E. (1964). Outlines of a theory of the light sense (L. Hurvich \& D. Jameson, Trans.). Cambridge, MA: Harvard University Press. (Original work published 1874)

JAMESON, D., \& HURVICH, L. (1989). Essay concerning color constancy. Annual Review of Psychology, 40, 1-22.

JUDD, D. (1951). Colorimetry and artificial daylight. Proceedings of the 12th Session of the CIE, Stockholm, 1, 11.

JudD, D., MAcAdAM, D., \& WYSzecKI, G. (1964). Spectral distribution of typical daylight as a function of correlated color temperature. Journal of the Optical Society of America, 54, 1031-1040.

KATZ, D. (1935). The world of color. (R. MacLeod \& C. Fox, Trans.). London: Kegan Paul, Trench \& Trubner. (Original work published 1911)

KoFFKA, K. (1935). Principles of Gestalt psychology. New York: Harcourt, Brace.

LAND, E. (1977). The Retinex Theory of color vision. Scientific American, 237, 108-128.

LAND, E. (1986). Recent advances in Retinex Theory. Vision Research, 26, 7-21.

LEeUWEnBerG, E. (1982). The perception of assimilation and brightness contrast as derived from code theory. Perception \& Psychophysics, 32, 345-352.

MCCANN, J., MCKeE, S., \& TAYLOR, T. (1976). Quantitative studies in Retinex Theory. Vision Research, 16, 445-458.

Punengorgh, F. (1987). Kleurgeheugen [Color memory]. Unpublished master's thesis, University of Nijmegen, Nijmegen, The Netherlands.

Reid, R., \& Shapley, R. (1988). Brightness induction by local contrast and the spatial dependence of assimilation. Vision Research, 28 , 115-132.

Tipltz Blackwell, K., Buchsbaum, G. (1988). The effect of spatial and chromatic parameters on chromatic induction. Color Research \& Application, 13, 166-173.
Troost, J., \& DE WeERT, C. (1991). Surface reflectance and human color constancy. Psychological Review, 98, 143-145.

UChiKaWA, H., UChIKAWA, K., Boynton, R. (1989). Influence of achromatic surrounds on categorical perception of surface colors. Vision Research, 29, 881-890.

VAlBerg, A., \& LANGe-MALECK1, B. (1990). "Colour constancy" in Mondrian patterns: A partial cancellation of physical chromaticity shifts by simultaneous contrast. Vision Research, 30, 371-380.

VAN Trigt, C. (1990). Smoothest reflectance functions: I. Definition and main results. Joumal of the Optical Society of America A, 7, 18911904.

von Kries, J. (1905). Die Gesichtsempfindungen. In W. Nagel (Ed.), Handbuch der Physiologie des Menschen (Vol. 3, pp. 109-282). Braunschweig.

Vos, J. (1978). Colorimetric and photometric properties of a $2^{\circ}$ fundamental observer. Color Research \& Application, 3, 125-128.

VOS, J., \& WALRAVEN, P. (1970). On the derivation of the foveal receptor primaries. Vision Research, 11, 795-818.

WERnER, J. S., W WALRAVEN, J. (1982). Effect of chromatic adaptation on the achromatic locus: The role of contrast, luminance and background color. Vision Research, 22, 929-943.

Wyszecki, G., Stiles, W. (1967). Color science. New York: Wiley.

\section{APPENDIX}

All chromaticities under the test illuminants were calculated by applying a "von Kries transformation" to the chromaticities in the standard illuminant condition. We chose the von Kries method because we wanted to avoid the manipulation and construction of illuminant and reflectance spectra that are incorporated in models such as described by Dannemiller (1989) and van Trigt (1990). Specifically, by putting severe constraints on the infinite set of possible reflectance functions that give the same tristimulus values under a certain illuminant (i.e., metameric reflectances), these models describe a one-to-one relation between tristimulus values and reflectance spectra for any specified illuminant spectrum. Because an illuminant spectrum is required as input, these models are restricted to make predictions under known illuminant spectra. Published tables (e.g., Bartleson, 1980; Wyszecki \& Stiles, 1967) and methods to obtain these spectra (Judd, MacAdam, \& Wyszecki, 1964) cover only phases of daylight or artificial approximations of daylight, so another method is required if illuminants are more arbitrarily chosen (i.e., not restricted to represent daylight, as, for example, in this paper or in McCann, McKee, \& Taylor, 1976). The von Kries transformation is a method that makes good predictions, independent of the exact illuminant and reflectance spectra. Furthermore, it can be argued that the predictions of spectra models cannot be empirically discriminated from von Kries predictions (see Dannemiller, 1989, p. 259).

To apply the von Kries transformation, each chromaticity under the standard illuminant had to be converted to receptor responses. To obtain receptor responses, using Vos-Walraven primaries (Vos \& Walraven, 1970) as tabulated by Vos (1978), a number of intermediate conversions are required. First, the $u, v$ coordinates of the target (or surround) colors have to be transformed to $x, y$ coordinates:

$$
\begin{aligned}
& x=3 u /(2 u-8 v+4), \\
& y=2 v /(2 u-8 v+4) .
\end{aligned}
$$

Judd's (1951) modification of the $x, y$ coordinates is now applied to obtain $x^{\prime}, y^{\prime}$ coordinates and luminance $Y^{\prime}$. This relation is given by 
$x^{\prime}=(1.0271 x-0.00008 y-0.00009) /(0.03845 x+0.01496 y+1)$,

$y^{\prime}=(0.00376 x+1.0072 y+0.00764) /(0.03845 x+0.01496 y+1)$, $Y^{\prime}=Y$.

It should be noted that $Y^{\prime}$ does not exactly correspond to $Y$. However, as long as stimuli are not in the extreme saturated blue region of the $x, y$-chromaticity diagram, the differences are negligible.

For a stimulus with chromaticity coordinates $x^{\prime}, y^{\prime}$, and luminance $Y^{\prime}$, the tristimulus values are given by

$$
\begin{aligned}
X^{\prime} & =\left(x^{\prime} / y^{\prime}\right) Y^{\prime}, \\
Y^{\prime} & =Y^{\prime}, \\
Z^{\prime} & =\left(\left(1-x^{\prime}-y^{\prime}\right) / y^{\prime}\right) / Y^{\prime}
\end{aligned}
$$

Following Vos (1978), $R, G$, and $B$ are given by

$$
\begin{aligned}
& R=0.1551646 X^{\prime}+0.5430763 Y^{\prime}-0.0370161 Z^{\prime}, \\
& G=-0.1551646 X^{\prime}+0.4569237 Y^{\prime}+0.0296946 Z^{\prime}, \\
& B=0.0073215 Z^{\prime} .
\end{aligned}
$$

Once receptor responses $R, G$, and $B$ of chromaticities of simulated patches under the standard illuminant are known, receptor responses $R^{\circ}, G^{\circ}$, and $B^{\circ}$ under one of the test illuminants can be derived by

$$
\begin{aligned}
& R^{\cdot}=a_{\mathrm{R}} R, \\
& G^{\cdot}=a_{\mathrm{G}} G, \\
& B^{\cdot}=a_{\mathrm{B}} B,
\end{aligned}
$$

in which von Kries coefficients $a$ are given by

$$
\begin{aligned}
& a_{\mathrm{R}}=R_{\mathrm{REF}} / R_{\mathrm{REF}}, \\
& a_{\mathrm{G}}=G_{\mathrm{REF}} / G_{\mathrm{REF}}, \\
& a_{\mathrm{B}}=B_{\mathrm{REF}} / B_{\mathrm{REF}},
\end{aligned}
$$

where $R_{\mathrm{REF}}, G_{\mathrm{REF}}$, and $\boldsymbol{B}_{\mathrm{REF}}$ stand for the standard illuminants and $R_{\text {REF }}, G_{\mathrm{REF}}$, and $B_{\mathrm{REF}}$ for the test illuminants. Both illuminants are assumed to have the same energy. Unfortunately, the von Kries adaptation model does not describe how to obtain the references themselves. However, there is general agreement to take a spatial average (e.g., Brill \& West, 1986; Land, 1986). Results of empirical studies suggest that a spatially weighted average should be used (Reid \& Shapley, 1988; Tiplitz Blackwell \& Buchsbaum, 1988). In fact, this was the motivation to choose the average color of both inner and outer rings of surrounding hexagons in the matching experiments equal to the background color that had the chromaticity of the illuminant.

Once $R^{*}, G^{*}$, and $B^{*}$ are obtained, one can find the $u, v$ coordinates by applying the reverse procedure. First,

$$
\begin{aligned}
& X^{\prime}=2.94483 R^{\cdot}-3.50013 G^{\circ}+29.08968 B^{\prime}, \\
& Y^{\prime}=1.00000 R^{\circ}+1.00000 G^{\circ}+1.00137 B^{\circ}, \\
& Z^{\prime}=136.6303 B^{\circ} .
\end{aligned}
$$

Then from tristimulus values to chromaticity coordinates $x^{\prime}, y^{\prime}$, and luminance $Y^{\prime}$,

$$
\begin{aligned}
& x^{\prime}=X^{\prime} /\left(X^{\prime}+Y^{\prime}+Z^{\prime}\right), \\
& y^{\prime}=X^{\prime} /\left(X^{\prime}+Y^{\prime}+Z^{\prime}\right), \\
& Y^{\prime}=Y^{\prime} .
\end{aligned}
$$

And to $x, y$, and $Y$,

$$
\begin{aligned}
x= & \left(1.00709 x^{\prime}+0.00008 y^{\prime}+0.00009\right) / \\
& \left(-0.03867 x^{\prime}-0.01537 y^{\prime}+1.03450\right), \\
y= & \left(-0.00347 x^{\prime}+1.02710 y^{\prime}-0.00785\right) / \\
& \left(-0.03867 x^{\prime}-0.01537 y^{\prime}+1.03450\right), \\
Y= & Y^{\prime} .
\end{aligned}
$$

And finally, from $x, y$ coordinates to $u, v$ coordinates with

$$
\begin{aligned}
& u=4 x /(-2 x+12 y+3), \\
& v=6 y /(-2 x+12 y+3) .
\end{aligned}
$$

(Manuscript received December 11, 1990; revision accepted for publication July 17, 1991.) 